Review Article

\title{
The Intervention Effect of Traditional Chinese Medicine on the Intestinal Flora and Its Metabolites in Glycolipid Metabolic Disorders
}

\author{
Sha Di $\mathbb{D}^{1},{ }^{1}$ Yitian Wang, ${ }^{1}$ Lin Han $\mathbb{D},{ }^{1}$ Qi Bao, ${ }^{1}$ Zezheng Gao $\mathbb{D},{ }^{1}$ Qing Wang, \\ Yingying Yang $\mathbb{D},{ }^{1}$ Linhua Zhao $\mathbb{D},{ }^{1}$ and Xiaolin Tong $\mathbb{D}{ }^{1,2}$ \\ ${ }^{1}$ Department of Endocrinology, Guanganmen Hospital, China Academy of Chinese Medical Sciences, Beijing 100054, China \\ ${ }^{2}$ Shenzhen Hospital, Guangzhou University of Chinese Medicine, Guangzhou 518034, China \\ Correspondence should be addressed to Linhua Zhao; melonzhao@163.com and Xiaolin Tong; tongxiaolin@vip.163.com
}

Received 30 December 2018; Revised 31 March 2019; Accepted 24 April 2019; Published 4 June 2019

Academic Editor: Fatima Martel

Copyright (c) 2019 Sha Di et al. This is an open access article distributed under the Creative Commons Attribution License, which permits unrestricted use, distribution, and reproduction in any medium, provided the original work is properly cited.

\begin{abstract}
Metabolic syndrome (MS), which includes metabolic disorders such as protein disorder, glucose disorder, lipid disorder, and carbohydrate disorder, has been growing rapidly around the world. Glycolipid disorders are a main type of metabolic syndrome and are characterized by abdominal obesity and abnormal metabolic disorders of lipid, glucose, and carbohydrate utilization, which can cause cardiovascular and cerebrovascular diseases. Glycolipid disorders are closely related to intestinal flora and its metabolites. However, studies about the biological mechanisms of the intestinal flora and its metabolites with glycolipid disorders have not been clear. When glycolipid disorders are treated with drugs, a challenging problem is side effects. Traditional Chinese medicine (TCM) and dietary supplements have fewer side effects to treat it. Numerous basic and clinical studies have confirmed that TCM decoctions, Chinese medicine monomers, or compounds can treat glycolipid disorders and reduce the incidence of cardiovascular disease. In this study, we reviewed the relationship between the intestinal flora and its metabolites in glycolipid metabolic disorders and the effect of TCM in treating glycolipid metabolic disorders through the intestinal flora and its metabolites. This review provides new perspectives and strategies for future glycolipid disorders research and treatment.
\end{abstract}

\section{Introduction}

Metabolic syndrome (MS) comprises a cluster of metabolic disorders such as protein disorder, glucose disorder, lipid disorder, and carbohydrate disorder. Glycolipid metabolic disorders are the main type of MS and are characterized by abdominal obesity and abnormal metabolic disorders of lipids and carbohydrates in blood, including T2DM, obesity, hyperlipidemia, and insulin resistance. Glycolipid metabolic disorders are more complicated, because of multifactorial interaction, than single factor metabolic abnormalities and are the primary risk factors for cardiovascular and cerebrovascular diseases [1]. Therefore, a single intervention such as only reducing hyperglycemia or hypertension cannot effectively regulate multiple metabolic disorders. China Experts on Type 2 Diabetes with Dyslipidemia, published in 2017, reported that $42 \%$ T2DM patients have dyslipidemia, and the overall compliance rate is only $12 \%$ among patients treated with drugs [2]. Exploring an integrated prevention strategy has always been a problem for the medical community.

The intestinal flora consists of approximately 1014 bacteria that exist primarily in the large intestine. Intestinal flora has an important role in a host's physiology such as modulation of immunity development, inflammation, and energy metabolism. Intestinal flora's imbalance can contribute to diseases development, including obesity, hyperlipemia, T2DM, cardiovascular disease, irritable bowel syndrome, liver diseases, and skin diseases [3]. The resident microbiota produces a multitude of metabolites, which are employed to regulate cellular function [4]. Intestinal flora with its metabolites may provide us a new sight to explore the possible mechanisms of diseases. It may also identify many potential diagnostic and therapeutic targets. Ze-Hua Zhao reported that intestinal flora's metabolites, as mediators in the gut-liver axis, reveal 
the vital role of host-diet-microbiota-metabolite interactions in nonalcoholic fatty liver disease (NAFLA) [5]. The association of intestinal flora's metabolites from carbohydrate fermentation and protein fermentation in NAFLA, insulin resistance, and T2DM, has been reviewed by Emanuel E. Canfora [6].

$16 \mathrm{~S}$ ribosomal RNA (16S rRNA) gene sequencing and metagenomic sequencing methods have been employed in quantitative analysis of intestinal flora since the 1990s. The methods provide an effective approach to study intestinal flora's variation and function. 16S rRNA uses probes or primers designed to detect intestinal flora to specify which phylum, group, genus, or species it belonging to. Metagenomic sequencing uses taxonomically informative gene tags to target and amplify genomes to study intestinal flora's composite and function [7]. Metabolomics studies have been utilized to seek biomarkers and elucidate the pathophysiology and progression of diseases, such as insulin resistance [8], T2DM [9], and NAFLD [10], and predict pharmacokinetic profiles in vivo and evaluate the effectiveness and safety of drugs [11]. Connection of genomics and metabolomics provides us with more understanding of the relationship between gut microbiota and its metabolites and has also been applied in disease diagnostics and prognosis in precision medicine [12].

Traditional Chinese medicine (TCM) is an effective method for treating MS. Many basic and clinical studies have confirmed that Chinese decoctions, Chinese medicine monomers, or compounds can reduce lipid and carbohydrate in blood and decrease the incidence of cardiovascular disease. For people with abnormal glycolipid metabolic disorders, TCM has the clinical efficacy advantage of "multitarget treatment." Recent studies have demonstrated that TCM regulated lipid and carbohydrate by adjusting structure of disordered intestinal flora. For example, GeGenQilian decoction, Jiangtangtiaozhi formula, and other designed herbal formulas lowered carbohydrate level and regulated intestinal flora $[13,14]$. However, most current studies are limited to analyze the intestinal genomics and detect clinical indicators and do not explore the potential relationship between the effects of TCM on the intestinal flora and metabolites [1517]. A large gap remains regarding the exploration of changes in the biological mechanisms of the flora and its metabolites. This review describes the relationship of the intestinal flora and its metabolites in glycolipid metabolic disorders, and the mechanisms of TCM in treating glycolipid metabolic disorders through the intestinal flora and its metabolites.

\section{The Relationship of Intestinal Flora and Its Metabolites in Glycolipid Metabolism}

The intestinal flora has an important role in the pathophysiology of glycolipid metabolic diseases such as obesity and T2DM [18-20]. Patients with abnormal glycolipid metabolism have decreased intestinal bacterial diversity and gene richness. Transplantation of intestinal flora from obese patients can cause obesity in sterile mice, which suggests that the intestinal flora disorder can cause obesity and metabolic diseases [19]. In addition, the inoculation of the Enterobacter cloacae B29 strain, which was isolated from the stool of severe obese patients, into sterile mice can cause severe obesity and insulin resistance [21]. Compared with healthy people, patients with T2DM had a moderate imbalance in the intestinal flora $[22,23]$. An analysis of the gut flora of 292 Danish individuals also showed that individuals with low microbial abundance often had more proinflammatory bacteria, and most of them had insulin resistance, high triglycerides level, and had a higher risk of T2DM [24]. To date, studies have demonstrated that the structural imbalance in the intestinal flora primarily affects the metabolism of glycolipid in the body, which includes the metabolism of short-chain fatty acids (SCFAs), bile acids (BAs), choline, amino acids, and other metabolites.

2.1. Fatty Acids Metabolism and the Intestinal Flora. Fatty acids associated with energy metabolism, lipid metabolism, and cell membrane's constituents also act as signal molecules. Abnormal fatty acids metabolism leads to metabolic diseases such as T2DM, nonalcoholic hepatic steatosis, hyperlipemia, and obesity.

2.1.1. SCFAs Metabolism and the Intestinal Flora. SCFAs absorbed in the colon are major metabolites produced by anaerobic gut microbiota through the fermentation of indigestible carbohydrates such as polysaccharides, oligosaccharides, and fiber [25]. The SCFAs are produced in the colonic lumen and are transported across the epithelium by diffusion. When transported into colonic tissue, they are primarily converted by the colonic epithelium into lipids or ketone bodies such as $\beta$-hydroxybutyrate or acetoacetate [26].

SCFAs, which primarily consist of acetate, propionate, and butyrate (in a ratio of approximately 60:20:20 in the colon) [27], are thought to play a vital role in metabolism, inflammation, immune homeostasis, neural pathways, and cancer [28-31]. As for glycolipid metabolism, SCFAs can provide energy for various human tissues to maintain intestinal epithelial cell function, glucose homeostasis, and insulin sensitivity, which affects glucose, lipid, and cholesterol metabolism. At the same time, SCFAs used as substrates for gluconeogenesis also contribute to T2DM, obesity, hyperlipidemia, and other metabolic diseases by increasing a host's energy harvest [32].

SCFAs regulate glycolipid metabolism and are substrates that bind to G protein-coupled receptors (GPRs), including GPR41 (i.e., free fatty acid receptor 3 [FFAR3]), GPR43 (i.e., free fatty acid receptor 2 [FFAR2]), and GPR109A (i.e., hydroxycarboxylic acid receptor 2 [HCA2]), which are associated with inflammation, immunity, and cancer. Gut microbiota promotes adiposity and body weight via GPR41. However, SCFAs may prevent obesity by activating GPR43 [33]. GPR43 is associated with metabolic activity of the gut microbiota with host body energy homoeostasis processes, such as glycolipid metabolism, via suppressing insulin signaling in adipocytes [34]. Firmicutes, Bacteroidetes, and Actinobacteria are the primary phyla and Butyricicoccus, Blautia, and Phascolarctobacterium are the primary genera in the gut microbiota that have an important role in SCFAs metabolism $[35,36]$. In addition, SCFAs can 
be produced by bacteria in the genera Bacteroides, Bifidobacterium, Propionibacterium, Eubacterium, Lactobacillus, Clostridium, Roseburia, Lachnospira, and Prevotella [37, 38]. SCFAs are positively associated with Prevotella, Alistipes, and Barnesiella and negatively associated with Bacteroides and Enterococcus. Prevotellaceae bacteria are enriched in obesity [39]. Lachnospira, as SCFA producer, has a negative correlation with body weight [37]. Fecal SCFA level showed negative relation with bacterial diversity, and 70 unique microbial taxa had relationship with at least one SCFAs (acetate, butyrate, or propionate). Jacobo de la Cuesta-Zuluaga studied the relationship of fecal SCFAs, gut microbiota, gut permeability, and cardiometabolic outcomes, including obesity and hypertension in 441 community-dwelling adults. High fecal SCFAs, obesity, and cardiometabolic disease risk factors represented higher abundance of several disease-associated microbiota, including Enterobacter hormaechei, Haemophilus parainfluenzae, Streptococcus, and SMB53. Lower fecal SCFAs showed higher abundance of some beneficial microbes. Christensenellaceae, Methanobrevibacter, and Oscillospira had relationship with lower BMI, weight. Alistipes and Bacteroides associated with decreased obesity [40].

Acetate and propionate are primarily produced by the Bacteroidetes phylum bacteria, and butyrate is primarily produced by the Firmicutes phylum bacteria [41]. However, the gut microbiota has a complicated relationship with the SCFAs. For example, Murphy et al. showed that Firmicutes, Bacteroidetes, and Actinobacteria phyla bacteria were not associated with energy harvest and that the fecal SCFAs levels and fecal energy content in high-fat diet- (HFD-) fed mice were also not associated [42]. Acetate, the predominant SCFAs, is produced by anaerobic intestinal microbiota such as the Firmicutes phylum, Bacteroides (e.g., Bacteroides thetaiotaomicron), Proteobacteria (e.g., Desulfovibrio piger), Bifidobacterium, and Clostridia [43]. Acetate can strengthen tight junctions of epithelial cells with mediation a Bifidobacterium-induced improvement in the intestinal barrier against bacterial endotoxin [44]. Acetate provides energy for a host and is adsorbed by tissues involved in the synthesis of cholesterol, long-chain fatty acids, glutamine, and glutamate [45]. Acetate is positively associated with the phylum Tenericutes and the families Christensenellaceae, unclassified Clostridiales, Peptococcaceae, and Clostridiaceae. And, it is negatively associated with the genera Blautia Oscillospira [46], unclassified family in Clostridiales, Defluviitaleaceae, and Porphyromonadaceae in HFD-induced obesity [47].

Propionate and butyrate lower than acetate inhibit food intake [48]. Propionate and butyrate formation is associated with Lachnospiraceae, Ruminococcaceae, and Negativicutes (all members of the phylum Firmicutes) and the phyla Verrucomicrobia and Bacteroidetes [49]. The abundance of Bacteroidetes is positively associated with propionate and butyrate. Butyrate provides energy for colonic epithelial cells and suppresses inflammation, which is primarily caused by lipopolysaccharides [50]. 50\% propionate, as a precursor for gluconeogenesis, is used as the substrate for hepatic gluconeogenesis [51]. Propionate produced by gut microbiota prevented adipose tissue accumulation in lean twin transplanted mice [52]. Propionate and butyrate decrease the incidence of diet-induced obesity by regulating gut hormone release [48].

Butyrate produced by gut microbiota primarily provides energy for colonic cells [53] and is primarily produced by Clostridium clusters $I V$ and XIVa species of the genera Eubacterium and Butyrivibrio in the phylum Firmicutes [26]. Butyrate enters the mitochondria of colonocytes and undergoes $\beta$-oxidation to acetyl-CoA, which participates in the tricarboxylic acid (TCA) cycle. The level of butyrate decreased in HFD-induced rats [54]. Propionate and butyrate stimulate the release of peptide YY (PYY) and the hormones glucagon-like peptide 1 (GLP-1) from intestinal L-cells, and thereby regulate food intake and glucose tolerance [55]. PYY produced by GPR41 is an enteroendocrine cell-derived hormone such as the incretins GLP-1 and glucose-dependent insulinotropic polypeptide, which normally inhibits gut motility and thereby absorbs more energy from feces, decreases the intestinal transit rate, and increases the harvest of energy from the diet [56]. GPR41 plays a vital role in the butyrate stimulation of GLP-1. GPR41-deficient mice have a normal body weight and glucose homeostasis [48]. Increased butyrate excretion was in positively correlation with the abundances of Faecalibacterium prausnitzii, Roseburia faecis, and other Clostridiales, known as butyrate-producers, as well as Enterobacter hormaechei, Haemophilus parainfluenzae, and Streptococcus, winch were unknown for fermentation capacity in obesity and hypertension of 441 communitydwelling adults. Almost microbiota significantly associated with butyrate excretion was also connected with acetate or propionate excretion [40].

In addition, SCFAs stimulate GLP-2 in the ileum, which decreases gut barrier permeability and lipopolysaccharide level. Propionate is primarily delivered and used by the liver, which has an important role in gluconeogenesis. Butyrate, as substrate, binds to GPR109a and is associated with the gut microbiota. Gpr109a is not expressed in the colons of germ-free and antibiotic-treated mice. The drug tributyrin, which can be metabolized into butyrate, induces GPR109a expression in antibiotic-treated animals [26].

2.1.2. The Metabolism of Other Fatty Acids and the Intestinal Flora. Unsaturated fatty acids can inhibit lipotoxicity caused by palmitic acid (16:0) [57]. Saturated fatty acids are risk factors for developing obesity and its associated disorders. Polyunsaturated fatty acids and monounsaturated fatty acids can inhibit the hepatic steatosis process [58]. Free fatty acids (FFAs) are associated with obesity, inflammation, altered glucose homeostasis, and cardiovascular disease. The genus Akkermansia is negatively associated with FFAs, and an alteration in FFAs levels is associated with imbalanced levels of Akkermansia and Lactobacillus [59]. Patients with MS have higher levels of saturated fatty acids, FFAs, monounsaturated fat, and trans fat (i.e., trans-fatty acids) [60, 61].

Gut microbiota lowers fatty acids oxidation by inhibiting the production of adenosine monophosphate kinase, which has a vital role in energy metabolism. Increased fatty acids oxidation enhances the cellular energy status, and increased insulin sensitivity decreases the glycogen level. Germ-free mice have increased adenosine monophosphate kinase levels 
in the liver and skeletal tissues, and enhanced fatty acids oxidation [62]. Monounsaturated fatty acids and saturated fatty acids in fasting serum are associated with the increased abundance of Blautia and Dorea bacteria, and decreased levels of Coprococcus and Peptococcaceae bacteria [46, 61].

2.2. Bile Acids Metabolism and the Intestinal Flora. Primary BAs are the main ingredient of bile and are synthesized from cholesterol in the liver through two major bile acid synthetic pathways: the classic pathway and the alternative pathway [63]. These acids are collected in the gall bladder and further released into the duodenum after a meal through bile. This action promotes the metabolism of dietary lipids and lipid-soluble vitamins. The duodenum, jejunum, and proximal ileum have high concentrations of bile salts. The proximal ileum digests and absorbs fat, and the distal ileum absorbs bile salts. Small bowel microbes promote bile salt metabolism via deconjugation and hydroxy group oxidation. More than $95 \%$ bile salts are absorbed by the ileum and cycled into the liver-a process called enterohepatic circulation. The remaining $5 \%$ of BAs escape the enterohepatic circulation are converted into secondary BAs, which include mostly deoxycholic acid (i.e., $3 \alpha, 12 \alpha$-dihydroxy- $5 \beta$-cholan-24-oic acid) and lithocholic acid (i.e., $3 \alpha$-hydroxy- $5 \beta$-cholan-24oic acid). These acids enhance toxicity and are more easily absorbed by the intestine and gut microbiota, especially the Firmicutes phyla, with bacterial enzymes in the large intestine $[32,64]$.

The BAs, as signaling molecules, regulate BAs metabolism, lipid utilization, glucose utilization, energy, drug metabolism, inflammation, detoxification, and immunity by activating nuclear receptors. These nuclear receptors include the Farnesoid X receptor (FXR), cell surface GPRs, constitutive androstane receptor, vitamin $\mathrm{D}$ receptor, and pregnane $\mathrm{X}$ receptor. Cell surface GPRs also include the $\mathrm{G}$ proteincoupled bile acids receptors (e.g., TGR5 and GPBAR-1) [6365]. FXR, an intracellular BAs sensor and transcriptional regulator, regulates BAs homeostasis and lipid homeostasis. Serum BAs, cholesterol, triglycerides, hepatic cholesterol, triglycerides, and a proatherogenic serum lipoprotein profile are dramatically increased in FXR/BAR-null mice than in wild-type mice [66]. Intestine-specific activation of FXR can alleviate obesity and metabolic deterioration phenotype in mice and can promote body heat production and white fat browning [67]. TGR5 activated by BAs increases energy expenditure and improves obesity caused by diet. In addition, TGR5 signaling promotes GLP-1 release, which improves liver and pancreatic function and glucose tolerance in obese mice [68].

Gut microbiota regulate the biotransformation of BAs by deconjugation, dehydroxylation, and reconjugation, which change BAs composition and modulate FXR and TGR5 signaling. There are four gut microbiota phyla in the small intestine, including Clostridium, Bacteroides, Lactobacillus, Bifidobacterium, and Enterococcus, which secrete bacterial bile salt hydrolase enzyme that deconjugates primary BAs in the small intestine [69]. Some gut microbiota is involved in BAs metabolism. For example, Bacteroides, Bifidobacterium, Clostridium, Lactobacillus, and Listeria are involved in deconjugation. Bacteroides, Clostridium, Escherichia, Eggerthella, Eubacterium, Peptostreptococcus, and Ruminococcus are involved in oxidation and epimerization. Clostridium, and Eubacterium are involved in 7-dehydroxylation. Bacteroides, Eubacterium, and Lactobacillus are involved in esterification. And Clostridium, Fusobacterium, Peptococcus, and Pseudomonas are involved in desulfation [70]. Lactobacillus can produce bile salt hydroxylase [71]. Fecal secondary BAs are associated with Clostridium cluster XVIa bacteria [72].

Gut microbiota play a role in transforming primary BAs, which is involved in lipid and glucose metabolism [73]. Mice treated with tempol, which can prevent obesity, had increased the level of Bacteroidetes bacteria and decreased the level of Firmicutes and Lactobacillus bacteria, and synthesized more BAs that regulated the FXR [71]. Rats injected with irinotecan (i.e., CPT-11) have disturbance in the intestinal microbiota, which results in quantitative and qualitative changes in the BAs. Serum $12 \alpha$-hydroxylated BAs and their conjugates increased and were correlated with insulin resistance in diabetic patients [74]. Cholic acid (i.e., $3 \alpha, 7 \alpha, 12 \alpha$-trihydroxy$5 \beta$-cholan-24-oic acid) and chenodeoxycholic acid (i.e., $3 \alpha$, $7 \alpha$-dihydroxy-5 $\beta$-cholan-24-oic acid) are primary BAs and synthesized in the liver. They conjugate with glycine or taurine and convert the molecules into conjugated BAs or bile salts. The levels of deoxycholic acid and taurodeoxycholic acid were significantly increased in plasma and liver tissues, and deoxycholic acid also raised in intestinal tissues and feces in obesity rat, which were connection with enhancement of genera Blautia, Coprococcus, Intestinimonas, Lactococcus, Roseburia, and Ruminococcus $[75,76]$.

2.3. Choline Metabolism and the Intestinal Flora. Choline is a water-soluble nutrient that is essential for human life [77]. It is also a vital ingredient of cell membranes, mitochondrial membranes, and acetylcholine, which play a role in certain physiological activities such as lipid metabolism, glucose metabolism, signaling through lipid second messengers, enterohepatic circulation of bile and cholesterol, and mitochondrial bioenergetics [78]. Compared with the normal population, T2DM patients have dysfunctions in the metabolism of choline, glucose, lipids, and amino acids. Decreased bioavailability of choline can increase the incidence of NAFLA and can alter glucose metabolism [73]. Decreased serum level of choline in diabetic patients is associated with the gut microbiota [79].

Firmicutes, Proteobacteria phyla bacteria and other anaerobic gut microbiota produce enzymes that convert dietary choline into trimethylamine (TMA), which cannot be synthesized by humans. Trimethylamine is an intermediate in the conversion of choline to betaine, methylamine, dimethylamine (DMA), and trimethylamine N-oxide (TMAO), the formation of which is associated with intestinal bacteria action $[69,80]$. Flavin monooxygenases 1 and 3 in the liver can metabolize TMA to TMAO, which is associated with impaired renal function, colorectal cancer, diabetes, and cardiovascular disease. Mice fed HFD with $0.2 \%$ TMAO had higher fasting insulin level and homeostasis model assessment-estimated insulin resistance and impaired 
glucose tolerance associated with the hepatic insulin signaling pathway and adipose tissue inflammation [81]. The following bacteria from two phyla (i.e., Firmicutes and Proteobacteria) and six genera showed significant choline consumption and TMA accumulation: Anaerococcus hydrogenalis, Clostridium asparagiforme, Clostridium hathewayi, Clostridium sporogenes, Escherichia fergusonii, Proteus penneri, Providencia rettgeri, and Edwardsiella tarda [77]. Fecal choline connected with Bacteroides and negatively with Prevotella and Clostridium, the secondary BAs deoxycholic acid connected with Bacteroides, Erysipelotrichaceae incertae sedis, and Enterococcus, but negatively with Prevotella, Barnesiella, and Alistipes in mice. Fecal SCFAs connected with Prevotella, Barnesiella, and Alistipes and negatively with Bacteroides, Erysipelotrichaceae incertae sedis and Enterococcus in mice [76].

The leave of trimethylamine $N$-oxide is negatively associated with Peptococcaceae, Prevotella, and Faecalibacterium prausnitzii and is significantly and positively associated with Allobaculum, Candidatus Arthromitus, or Lachnospiraceae [82]. Reduced levels of Faecalibacterium prausnitzii have been associated with obesity, diabetes, and several immunerelated diseases [46]. Plasma TMA and TMAO levels are positively correlated with Clostridiales, Ruminococcus, and Lachnospiraceae bacteria in female mice [83].

2.4. Amino Acids Metabolism and the Intestinal Flora. Gut bacteria can change bioavailability of amino acids via utilizing certain amino acids originating from alimentary proteins and endogenous proteins and can supply amino acids for a host. Amino acids support growth and survival of gut bacteria and regulate energy and protein homeostasis. Elevated level of amino acids, particularly branched-chain and aromatic amino acids (e.g., phenylalanine and tyrosine), promotes the development of insulin resistance and T2DM.

Certain amino acids, as precursors for the synthesis of SCFAs, are liberated by gut microbiota and are involved in the development of obesity. Clostridium, Bacillus, Lactobacillus, Streptococcus, and Proteobacteria in the small intestine and Clostridia, Peptostreptococcus in large intestine can ferment amino acids. This process is associated with protein digestion and subsequently with amino acid absorption. Colonic bacteria primarily use certain amino acids as substrates such as the branched-chain amino acids (BCAAs), lysine, arginine, and glycine. They produce metabolic end-products such as SCFAs and branched-chain fatty acids such as valerate, isobutyrate, and isovalerate. These bacterial metabolites can affect signaling pathways in epithelial cells and in the mucosal immune system, and thereby influence epithelial physiology. The BCAAs, aromatic amino acids (e.g., tyrosine), and other amino acids (e.g., alanine, lysine, and methionine) are positively associated with Prevotella, Alistipes, and Barnesiella, but are negatively associated with Bacteroides and Enterococcus. In addition, Prevotellaceae is significantly enriched in obesity [84].

2.4.1. Branched-Chain Amino Acids and the Intestinal Flora. BCAAs include leucine, isoleucine, and valine. BCAAs are involved in the synthesis of protein, alanine, glutamine, and glucose metabolism and oxidation [85]. Plasma BCAAs are increased in patients with metabolic disorders such as high fasting glucose level and insulin resistance [86]. Isoleucine can increase insulin-independent glucose uptake in vitro and improve glucose metabolism and oxidation. In fasted rats, oral isoleucine reduced the plasma glucose level and increased muscle glucose uptake, which was correlated with decreased messenger ribonucleic acid (mRNA) levels of phosphoenolpyruvate carboxykinase, glucose-6-phosphatase (G6Pase), and G6Pase in isolated hepatocytes [87]. BCAAs are negatively associated with abundance of Christensenellaceae and are positively associated with Blautia [46].

2.4.2. Other Amino Acids and the Intestinal Flora. Prediabetic patients reduced levels of amino acids such as $\mathrm{L}$ lysine, L-threonine, betaine, isovaleraldehyde, 2-ketobutyric acid, 2-pyrroloylglycine, and abnormal glutamine, glycine, serine, and threonine [88]. The level of 2-Ketobutyric acid is correlated with glycine, methionine, valine, leucine, serine, threonine and isoleucine, which are associated with diabetes. Some amino acids such as 2-ketobutyric acid, glycine, serine, and threonine have an important role in the TCA cycle [3]. Individuals with obesity or visceral obesity have increased levels of lysine, tryptophan, cystine, and glutamate, and decreased levels of asparagine, citrulline, glutamine, glycine, and serine.

The fasting and 2-hour plasma glucose levels and the homeostasis model assessment of insulin resistance are positively associated with valine, glutamate, and tyrosine levels, but negatively associated with citrulline, glutamine, and glycine levels [89]. The Photobacterium genus was negatively associated with alanine, glycine, taurine, whereas Coprococcus 3 and Vibrio genera were positively associated with citrulline [90]. Tryptophan, which is the amino acid precursor for the signaling molecule 5-hydroxytryptophan, is regulated by gut microbiota such as Bifidobacterium and Bacteroides fragilis [73]. Bacteroides thetaiotaomicron reduced the plasma glutamate level and improves diet-induced body weight gain and adiposity in mice. This finding suggests that glutamate is negatively associated with $B$. thetaiotaomicron [91].

Some metabolites are also altered in MS. Hippurate, 4-hydroxylphenylacetic acid, and phenylacetylglycine levels are increased and acetate, lactate levels are decreased in urine. These metabolites are associated with obesity and an alteration in gut microbiota [35]. Niacin metabolites such as nicotinuric acid and trigonelline, which are associated with gut microbiota, are connected with obesity and T2DM. Diabetic patients had lower level of trigonelline in association with a change in energy and tryptophan metabolism [69]. Allantoin, an oxidative product of uric acid, was higher in mice with HFD-induced hyperlipidemia, which was associated with oxidative stress $[92,93]$.

2.5. The TCA Cycle and the Intestinal Flora. The intermediates of TCA cycle include citrate, butanedioic acid, citrate, succinate, pyruvate, and cis-aconitate. These intermediates have a vital role in energy metabolism [94]. Pyruvate is an important metabolite in glycolysis and in TCA cycle. 
It converts pyruvate into acetyl-coenzyme A (acetyl-CoA), which enters TCA cycle. Reduced glycolysis may result in a higher level of glucose. Pyruvate is positively associated with Coriobacteriaceae and Blautia. Rats with HFD-induced hyperlipidemia increased levels of pyruvate and decreased levels of citrate, succinate, and cis-aconitate and caused dysfunction in glucose metabolism, insulin utilization, and energy metabolism. Increased fatty acid oxidation occurred in rats with hyperlipidemia, which might generate SCFAs or medium-chain fatty acids, and thereby reduced glucose metabolism, and changed energy consumption to lipid oxidation [95]. Decreased levels of pyruvate, citrate, succinate, fumarate, and 2-oxoglutarate and increased levels of pantothenic acid occurred in the serum of HFD-fed mice. In addition, the liver of HFD-fed mice decreased levels of the glycolysis end-products pyruvate and lactate, glutamate and decreased levels of the TCA cycle intermediates citrate, succinate, fumarate, malate, and oxaloacetate [96]. Increased TCA enhances the secretion of GLP-1, which is associated with the depletion of Firmicutes and Bacteroidetes bacteria [97]. The relationship of gut microbiota and its metabolites are summarized in Table 1.

\section{The Effect of TCM Treatment on the Intestinal Flora and Its Metabolites in Glycolipid Metabolism Diseases}

Experiments have shown that diet control, TCM treatment, and other methods can regulate the intestinal flora structure and its metabolites, reduce inflammatory factors, and relieve insulin resistance, obesity, metabolic disorders [98-100].

3.1. TCM Improves SCFAs and the Associated Enrichment of Bacteria. The abundance of SCFAs-producing bacteria in the intestines of patients with glycolipid disorders is decreased. Many Chinese medicines regulate glycolipid metabolism by enriching bacteria producing SCFAs. For example, Rhizoma Coptidis alkaloids regulated the structure of gut microbiota to alleviate hyperglycemia, which might help alleviate inflammation by increasing the level of intestinal SCFAs [101]. Xiexin Tang altered SCFA-producing and anti-inflammatory bacteria such as Adlercreutzia, Alloprevotella, and Barnesiella in T2DM [102]. Shenling Baizhu powder increased SCFAs-producing bacteria including Bifidobacterium and Anaerostipes in NAFLD rats [103]. Oral hydroxysafflor yellow $\mathrm{A}$, an active compound from the dried florets of Carthamus tinctorius L., increased SCFAs-producing bacteria, including genera Butyricimonas and Alloprevotella in obesity mice [104]. Huang-Lian-Jie-Du-Decoction enriched SCFAsproducing and anti-inflammatory bacteria, such as Parabacteroides, Blautia, and Akkermansia in T2DM rats [105]. JinQi Jiangtang tablets enriched Akkermansia spp., a bacteria producing SCFAs, and reduced Desulfovibrio in T2DM [106]. A water insoluble polysaccharide increased Lachnospiraceae and Clostridium in ob/ob mice with hepatic steatosis. Lachnospiraceae and Clostridium can produce butyrate [107]. A specifically designed herbal formula increased Faecalibacterium and Roseburia, which contained butyrate-producing bacteria. It also enriched SCFA-producing bacteria, including Gemmiger, and Coprococcus in T2DM [14]. Berberine increased SCFAs in feces and SCFAs-producing microbiota such as Blautia and Allobaculum in insulin-resistant rats [101]. And Berberine also enhanced SCFAs-producing bacteria such as Allobaculum, Blautia, Bacteroides, Butyricicoccus, and Phascolarctobacterium in rats with HFD-induced obesity [36].

3.2. TCM Improves BAs and Related Bacteria. TCM ingredients can affect genes expression, and consequently regulate FXR, or structure of intestinal flora to regulate the conversion of BAs in the liver and intestine. Resveratrol significantly increased the abundance of Lactobacillus and Bifidobacterium and increased the activity of intestinal bile acid hydrolase to promote the catabolism of BAs in the intestine, which promoted the efflux of fecal BAs. The synthesis of BAs in the liver was ultimately promoted [108]. Total saponins in Gynostemma have the pharmacological effects of regulating lipid metabolism. Gynostemma can regulate BAs metabolic pathways and bile acid layer, and its target may be the nuclear receptor FXR [109]. Pepper extract decreased lipid by regulating BAs in the intestines and liver. It promoted intestinal peristalsis, and increased ileum FXR gene expression, BAs level, and neutral steroid excretion. Meanwhile, it reduced BAs reflux in intestinal circulation, and accelerated cholesterol decomposition by lowering liver FXR expression [83]. Berberine prominently lowered BCAA-producing bacteria, including order Clostridiales, families Streptococcaceae, Clostridiaceae, and Prevotellaceae, and genera Streptococcus and Prevotella in HFD-fed mice with insulin resistance [110]. Epigallocatechin-3-gallate regulated bile acid signaling and enriched Akkermansia muciniphila in diet-increased obesity [111].

3.3. TCM Improves TMA/TMAO Levels and Related Bacteria. The compound of vinegar and olive oil (which contains 3, 3-dimethyl-1-butanol) can effectively increase SCFAs levels and block TMAO production in mice [112]. Resveratrol can reduce the level of TMA by remodeling the intestinal flora, thereby affecting lipid metabolism and preventing arteriosclerosis [108].

3.4. Effect of TCM on Other Metabolites. Many TCM ingredients act on amino acid metabolism and TCA, and participate in energy metabolism. Turmeric regulated fat production and decomposition pathways to lower blood lipids. Curcumin-treated mice had increased levels of acetate, leucine, isoleucine, valine, alanine, and TCA cycle intermediates (e.g., lemon acid salt, succinate, and cis-aconitate). Berberine is involved in glycolysis and the TCA cycle, amino acid metabolism, vitamin B6 metabolism, and secondary bile acid biosynthesis [113]. Gardenia jasminoides regulated metabolic perturbations in phenylalanine, tyrosine, and tryptophan biosynthesis, tryptophan metabolism, and purine metabolism [114]. Qijian mixture, consisting of Astragalus membranaceus, Ramulus euonymi, Coptis chinensis, and Pueraria lobata, changed related proteins and signaling pathways, including valine, leucine and isoleucine degradation 
TABLE 1: Metabolites of gut microbiota.

Metabolites

Fatty acids
Related gut microbiota

Phyla:

Firmicutes,

Bacteroidetes,

Actinobacteria,

Proteobacteria,

Tenericutes,

Verrucomicrobia

Class:

Clostridia

Order:

Clostridiales

Family:

Christensenellaceae,

Lachnospiraceae,

Ruminococcaceae,

Negativicutes,

Enterobacter hormaechei

Genus:

Akkermansia,

Lactobacillus,

Bacteroides,

Bifidobacterium,

Propionibacterium,

Eubacterium,

Lactobacillus,

Clostridium,

Roseburia,

Lachnospira,

Prevotella,

Butyricicoccus,

Blautia,

Phascolarctobacterium,

Butyrivibrio,

Faecalibacterium prausnitzii,

Streptococcus

Species:

Haemophilus parainfluenzae,

Coprococcus,

Peptococcaceae

Phyla:

Firmicutes,

Clostridium,

Bacteroides,

Lactobacillus,

Bifidobacterium,

Enterococcus

Genus:

Listeria,

Escherichia,

Eubacterium,

Peptostreptococcus,

Ruminococcus,

Fusobacterium,

Peptococcus,

Pseudomonas,

Blautia,

Coprococcus,

Intestinimonas,

Lactococcus,

Roseburia

Others:

Eggerthella
Provide energy to maintain intestinal epithelial cell function, regulate glucose homeostasis, lipid metabolism, and insulin sensitivity. 
TABLE 1: Continued.

Metabolites

Choline

Amino Acids
Related gut microbiota

Phyla:

Firmicutes,

Proteobacteria,

Bacteroides,

Prevotella,

Clostridium

Order:

Clostridiales

Genus:

Anaerococcus hydrogenalis,

Clostridium asparagiforme,

Clostridium hathewayi,

Clostridium sporogenes,

Escherichia fergusonii,

Proteus penneri,

Providencia rettgeri,

Edwardsiella tarda,

Erysipelotrichaceae incertae sedis,

Enterococcus,

Prevotella,

Barnesiella,

Alistipes,

Peptococcaceae,

Allobaculum,

Candidatus Arthromitus,

Ruminococcus

Family:

Faecalibacterium prausnitzii,

Lachnospiraceae

Phyla:

Proteobacteria

Class:

Clostridia

Family:

Prevotellaceae,

Christensenellaceae

Genus:

Bacteroides,

Clostridium,

Bacillus,

Lactobacillus,

Streptococcus,

Peptostreptococcus,

Prevotella,

Alistipes,

Barnesiella,

Enterococcus,

Blautia,

Photobacterium,

Coprococcus, Vibrio,

Bifidobacterium,

Bacteroidesfragilis,

Bacteroides thetaiotaomicron

$$
\text { Phyla: }
$$

Firmicutes,

Bacteroidetes

Family:

Coriobacteriaceae

Genus:

Blautia
Ingredient of cell membranes, mitochondrial membranes, and acetylcholine; play a role in certain physiological activities such as lipid metabolism, glucose metabolism.

\section{TCA}

Support growth and survival of gut bacteria; regulate energy and protein homeostasis; involved in the synthesis of protein, alanine, glutamine, and glucose metabolism and oxidation.
Have a role in energy metabolism, glucose metabolism, insulin utilization. 
TABLE 2: The effect of TCM treatment on intestinal flora and its metabolites.

\begin{tabular}{|c|c|c|}
\hline TCM treatment & Effect on intestinal flora & Effect on metabolites \\
\hline Xiexin Tang & $\begin{array}{c}\text { Altered Adlercreutzia, } \\
\text { Alloprevotella, Barnesiella }\end{array}$ & Altered SCFAs \\
\hline Shenling Baizhu powder & $\begin{array}{c}\text { Increased Bifidobacterium, } \\
\text { Anaerostipes }\end{array}$ & Increased SCFAs \\
\hline hydroxysafflor yellow A & $\begin{array}{c}\text { Increased Butyricimonas, } \\
\text { Alloprevotella }\end{array}$ & Increased SCFAs \\
\hline Huang-Lian-Jie-Du-Decoction & $\begin{array}{c}\text { Increased Parabacteroides, } \\
\text { Blautia, } \\
\text { Akkermansia }\end{array}$ & Increased SCFAs \\
\hline JinQi Jiangtang tablets & $\begin{array}{l}\text { Increased Akkermansia, } \\
\text { decreased Desulfovibrio }\end{array}$ & Increased SCFAs \\
\hline water insoluble polysaccharide & Increased Lachnospiraceae, Clostridium & Increased butyrate \\
\hline a specifically designed herbal formula & $\begin{array}{l}\text { Increased Faecalibacterium, Roseburia } \\
\text { Increased Gemmiger, Coprococcus }\end{array}$ & $\begin{array}{l}\text { Increased butyrate } \\
\text { Increased SCFAs }\end{array}$ \\
\hline \multirow[t]{2}{*}{ Berberine } & $\begin{array}{l}\text { Increased Blautia, Allobaculum, } \\
\text { Bacteroides, Butyricicoccus, } \\
\text { Phascolarctobacterium }\end{array}$ & Increased SCFAs \\
\hline & $\begin{array}{c}\text { Decreased Clostridiales, Streptococcaceae, } \\
\text { Clostridiaceae, Prevotellaceae, Streptococcus, } \\
\text { Prevotella }\end{array}$ & Decreased BCAA \\
\hline Resveratrol & Increased Lactobacillus, Bifidobacterium, & Increased BAs \\
\hline Epigallocatechin-3-gallate & Increased Akkermansia muciniphila & Regulated bile acid signaling \\
\hline Ferulic acid & $\begin{array}{c}\text { Altered the ratio of Firmicutes to } \\
\text { Bacteroidetes }\end{array}$ & Reduced indole-3-acetic acid \\
\hline
\end{tabular}

metabolism, aminoacyl-tRNA biosynthesis metabolism and alanine, aspartate, and glutamate metabolism pathways, with significantly enrichment of bacteroidetes [115]. Ferulic acid regulated gut microbiota's composition, including altering the ratio of Firmicutes to Bacteroidetes, and reduced indole3-acetic acid level in ApoE-/- mice fed on a high-fat diet [10]. The effect of TCM treatment on intestinal flora and its metabolites are summarized in Table 2.

\section{Summary}

As the number of obese people increases, glycolipid disorders become more common in the future. Glycolipid disorders have a significant impact on the quality of life and the financial resources of the public healthcare system. To date, treatment for T2DM with hyperlipidemia primarily relies on the use of hypoglycemic and lipid-lowering drugs. However, patients are increasingly concerned about potential toxicity and side effects and sometimes the clinical efficacy is unsatisfactory. Intensive studies on complementary and alternative medical therapies have increased the utility of these therapies for treating glycolipid metabolic diseases. Natural medicine, especially Chinese herbal medicine, constitutes a special medical system that is guided by TCM theory and provides effective treatment for glycolipid disorders.

Traditional Chinese herbal medicines are generally administered orally, and their digestion and absorption are inseparable from the intestinal flora. An increasing number of Chinese herbal ingredients have been proven to adjust an individual's intestinal flora, and thereby allow the flora to approximate that of a normal person. This trend is significantly associated with recovery from the diseases. Regulating the intestinal flora is an important way for Chinese medicines to take effect.

Among several metabolites, the SCFAs are most affected by Chinese medicine constituents such as berberine, Ganoderma lucidum, and resveratrol, which enrich SCFAsproducing bacteria, and increase intestinal SCFAs. The production of SCFAs can relieve insulin resistance and alleviate disorders of glycolipid metabolism. In addition, several traditional Chinese medicines can regulate BAs metabolism, upregulate FXR levels, increase insulin sensitivity, inhibit fat synthesis, and reduce serum triglyceride and cholesterol levels. These actions are closely associated with the regulation of the related gut microbiota. The level of TMA/TMAO is correlated with glycolipid metabolism and is an important marker and potential therapeutic target for cardiovascular disease. By intervening intestinal flora and its metabolites, TCM can achieve hypoglycemic and lipid-lowering effects and effectively reduce the incidence of cardiovascular events. In addition, TCM monomers are also widely involved in intestinal amino acid metabolism and in the TCA cycle.

In summary, TCM can regulate the body's glycolipid metabolism through the intestinal flora and its metabolites, and can achieve a therapeutic effect in people with glycolipid metabolic disorders. A Chinese medicine practitioner prescribes medicine, based on a patient's symptoms such as abdominal feeling, body shape, and stool shape. For patients with polydipsia, obesity, diabetes, and hyperlipidemia, TCM often eliminates digestion and promotes gastrointestinal 
motility, while relieving symptoms. TCM has a vital role in reducing sugar and fat levels. It can regulate glycolipid metabolism by altering the intestinal flora and its metabolites.

This review still has some limitations. Some conclusions are based on clinical trials with low levels of evidence, which causes biases. In addition, many studies lack evidence on the intestinal flora and its metabolites. The mechanism of TCM in treating glycolipid metabolic disorders needs further research. In the future, carefully designed, largescale, high-quality randomized controlled trials are needed to evaluate the effects of TCM on glycolipid metabolism. In addition, in-depth studies need to be conducted from the perspective of the flora to detect relevant targets in metabolic pathway and to clarify the biological mechanisms of Chinese medicine. Ideas need to be provided for preventing and treating glycolipid metabolism diseases.

\section{Conflicts of Interest}

The authors declared that they have no conflicts of interest.

\section{Authors' Contributions}

Dr. Sha Di and Yitian Wang contributed equally to this work.

\section{Acknowledgments}

This work was financially supported by the National Natural Science Foundation of China (no. 81430097) and China Academy of Chinese Medical Sciences (no. ZZ10-021).

\section{References}

[1] L. Ji, D. Hu, C. Pan et al., "Primacy of the 3B approach to control risk factors for cardiovascular disease in type 2 diabetes patients," American Journal of Medicine, vol. 126, no. 10, pp. 925.e11-925.e22, 2013.

[2] Lipid metabolism group and Chinese society of endocrinology, "Expert consensus on the prevention and treatment of type 2 diabetes mellitus with dyslipidemia in China (revised version in 2017)," Chinese Journal of Endocrinology and Metabolism, vol. 33, no. 11, 2017.

[3] M. Yu, H.-M. Jia, C. Zhou, Y. Yang, L.-L. Sun, and Z.-M. Zou, "Urinary and fecal metabonomics study of the protective effect of Chaihu-Shu-Gan-San on antibiotic-induced gut microbiota dysbiosis in rats," Scientific Reports, vol. 7, p. 46551, 2017.

[4] B. C. Lustri, V. Sperandio, and C. G. Moreira, "Bacterial chat: Intestinal metabolites and signals in host-microbiota-pathogen interactions," Infection and Immunity, vol. 85, no. 12, 2017.

[5] Z. H. Zhao, J. K. Lai, L. Qiao, and J. G. Fan, "Role of gut microbial metabolites in nonalcoholic fatty liver disease," Journal of Digestive Diseases, vol. 20, no. 4, pp. 181-188, 2019.

[6] E. E. Canfora, R. C. Meex, K. Venema, and E. E. Blaak, "Gut microbial metabolites in obesity, NAFLD and T2DM," Nature Reviews Endocrinology, vol. 15, no. 5, pp. 261-273, 2019.

[7] X. Huang, X. Fan, J. Ying et al., "Emerging trends and research foci in gastrointestinal microbiome," Journal of Translational Medicine, vol. 17, no. 1, p. 67, 2019.

[8] C. Papandreou, M. Bulló, M. Ruiz-Canela et al., "Plasma metabolites predict both insulin resistance and incident type 2 diabetes: a metabolomics approach within the Prevención con Dieta Mediterránea (PREDIMED) study," The American journal of clinical nutrition, vol. 109, no. 3, pp. 626-634, 2019.

[9] S. R. Khan, H. Mohan, Y. Liu et al., "Correction to: The discovery of novel predictive biomarkers and early-stage pathophysiology for the transition from gestational diabetes to type 2 diabetes," Diabetologia, vol. 62, no. 4, pp. 730-731, 2019.

[10] Y. Ma, K. Chen, L. Lv et al., "Ferulic acid ameliorates nonalcoholic fatty liver disease and modulates the gut microbiota composition in high-fat diet fed ApoE mice," Biomedicine pharmacotherapy, vol. 113, Article ID 108753, 2019.

[11] Z. Li, S. Zhao, Y. Yuan et al., "In vitro metabolic profiles of motolimod by using liquid chromatography tandem mass spectrometry: Metabolic stability, metabolite characterization and species comparison," Journal of Pharmaceutical and Biomedical Analysis, vol. 167, pp. 90-99, 2019.

[12] F. Brial, A. Le Lay, M.-E. Dumas, and D. Gauguier, "Implication of gut microbiota metabolites in cardiovascular and metabolic diseases," Cellular and Molecular Life Sciences, vol. 75, no. 21, pp. 3977-3990, 2018.

[13] J. Xu, F. Lian, L. Zhao et al., "Structural modulation of gut microbiota during alleviation of type 2 diabetes with a Chinese herbal formula,' The ISME Journal, vol. 9, no. 3, pp. 552-562, 2015.

[14] X. Tong, J. Xu, F. Lian et al., "Structural alteration of gut microbiota during the amelioration of human type 2 diabetes with hyperlipidemia by metformin and a traditional chinese herbal formula: a multicenter, randomized, open label clinical trial," mBio, vol. 9, no. 3, Article ID e02392-17, 2018.

[15] C. H. Zhang, J. Q. Sheng, S. Sarsaiya et al., "Anti-diabetic activities of scutellaria-coptis herb couple in type 2 diabetic KKAy mice and in RAW264.7 macrophages," Journal of Ethnopharmacology, pp. 202-214, 2019.

[16] W. Tang, X. Yao, F. Xia et al., "Modulation of the gut microbiota in rats by hugan qingzhi tablets during the treatment of highfat-diet-induced nonalcoholic fatty liver disease," Oxidative Medicine and Cellular Longevity, vol. 2018, Article ID 7261619, 14 pages, 2018.

[17] P. Bin, Z. Tang, S. Liu et al., "Intestinal microbiota mediates Enterotoxigenic Escherichia coli-induced diarrhea in piglets," BMC Veterinary Research, vol. 14, no. 1, p. 385, 2018.

[18] L. Zhao, "The gut microbiota and obesity: from correlation to causality," Nature Reviews Microbiology, vol. 11, no. 9, pp. 639647, 2013.

[19] P. J. Turnbaugh, R. E. Ley, M. A. Mahowald, V. Magrini, E. R. Mardis, and J. I. Gordon, "An obesity-associated gut microbiome with increased capacity for energy harvest," Nature, vol. 444, no. 7122, pp. 1027-1031, 2006.

[20] M. Vijay-Kumar, J. D. Aitken, F. A. Carvalho et al., "Metabolic syndrome and altered gut microbiota in mice lacking Toll-like receptor 5," Science, vol. 328, no. 5975, pp. 228-231, 2010.

[21] N. Fei and L. Zhao, "An opportunistic pathogen isolated from the gut of an obese human causes obesity in germfree mice," The ISME Journal, vol. 7, no. 4, pp. 880-884, 2013.

[22] J. Qin, Y. Li, Z. Cai et al., "A metagenome-wide association study of gut microbiota in type 2 diabetes," Nature, vol. 490, no. 7418, pp. 55-60, 2012.

[23] F. H. Karlsson, V. Tremaroli, I. Nookaew et al., "Gut metagenome in European women with normal, impaired and diabetic glucose control," Nature, vol. 498, no. 7452, pp. 99-103, 2013. 
[24] M. Diamant, E. E. Blaak, and W. M. de Vos, "Do nutrient-gutmicrobiota interactions play a role in human obesity, insulin resistance and type 2 diabetes?" Obesity Reviews, vol. 12, no. 4 , pp. 272-281, 2011.

[25] T. Arora and R. Sharma, "Fermentation potential of the gut microbiome: Implications for energy homeostasis and weight management," Nutrition Reviews, vol. 69, no. 2, pp. 99-106, 2011.

[26] S. Sivaprakasam, P. D. Prasad, N. Singh et al., "Benefits of short-chain fatty acids and their receptors in inflammation and carcinogenesis," Pharmacology therapeutics, vol. 164, pp. 144151, 2016.

[27] J. H. Cummings, "Short chain fatty acids in the human colon," Gut, vol. 22, no. 9, pp. 763-779, 1981.

[28] W. Ratajczak, A. Rył, A. Mizerski, K. Walczakiewicz, O. Sipak, and M. Laszczyńska, "Immunomodulatory potential of gut microbiome-derived short-chain fatty acids (SCFAs)," Acta Biochimica Polonica, vol. 66, no. 1, pp. 1-12, 2019.

[29] M. Rau, A. Rehman, M. Dittrich et al., "Fecal SCFAs and SCFA-producing bacteria in gut microbiome of human NAFLD as a putative link to systemic T-cell activation and advanced disease," United European Gastroenterology Journal, vol. 6, no. 10, pp. 1496-1507, 2018.

[30] Y. A. Yap and E. Mariño, "An insight into the intestinal web of mucosal immunity, microbiota, and diet in inflammation," Frontiers in Immunology, vol. 9, p. 2617, 2018.

[31] H. Zeng, S. Umar, B. Rust, D. Lazarova, and M. Bordonaro, "Secondary bile acids and short chain fatty acids in the colon: a focus on colonic microbiome, cell proliferation, inflammation, and cancer," International Journal of Molecular Sciences, vol. 20, no. 5, p. 1214, 2019.

[32] X. He, G. Ji, W. Jia, and H. Li, "Gut microbiota and nonalcoholic fatty liver disease: Insights on mechanism and application of metabolomics," International Journal of Molecular Sciences, vol. 17, no. 3, p. 300, 2016.

[33] M. Lyu, Y. Wang, G. Fan, X. Wang, S. Xu, and Y. Zhu, "Balancing herbal medicine and functional food for prevention and treatment of cardiometabolic diseases through modulating gut microbiota," Frontiers in Microbiology, vol. 8, p. 2146, 2017.

[34] I. Kimura, K. Ozawa, D. Inoue et al., "The gut microbiota suppresses insulin-mediated fat accumulation via the shortchain fatty acid receptor GPR43," Nature Communications, vol. 4, article 1829, 2013.

[35] P. Vernocchi, F. Del Chierico, L. Putignani et al., "Gut microbiota profiling: Metabolomics based approach to unravel compounds affecting human health," Frontiers in Microbiology, vol. 7, p. 1144, 2016.

[36] X. Zhang, Y. Zhao, J. Xu et al., "Modulation of gut microbiota by berberine and metformin during the treatment of high-fat diet-induced obesity in rats," Scientific Reports, vol. 5, Article ID 14405, 2015.

[37] S. Mayengbam, J. E. Lambert, J. A. Parnell et al., "Impact of dietary fiber supplementation on modulating microbiotahost-metabolic axes in obesity," The Journal of Nutritional Biochemistry, vol. 64, pp. 228-236, 2019.

[38] S. L. La Rosa, V. Kachrimanidou, F. Buffetto et al., "Woodderived dietary fibers promote beneficial human gut microbiota," mSphere, vol. 4, no. 1, 2019.

[39] H. Zhang, J. K. DiBaise, A. Zuccolo et al., "Human gut microbiota in obesity and after gastric bypass," Proceedings of the National Acadamy of Sciences of the United States of America, vol. 106, no. 7, pp. 2365-2370, 2009.
[40] J. de la Cuesta-Zuluaga, N. Mueller, R. Álvarez-Quintero et al., "Higher fecal short-chain fatty acid levels are associated with gut microbiome dysbiosis, obesity, hypertension and cardiometabolic disease risk factors," Nutrients, vol. 11, no. 1, Article ID E51, 2018.

[41] S. Macfarlane and G. T. Macfarlane, "Regulation of short-chain fatty acid production," Proceedings of the Nutrition Society, vol. 62, pp. 67-72, 2003.

[42] X. Zhang, Y. Zhao, M. Zhang et al., "Structural changes of gut microbiota during berberine-mediated prevention of obesity and insulin resistance in high-fat diet-fed rats," PLoS ONE, vol. 7, no. 8, Article ID e42529, 2012.

[43] A. Basson, A. Trotter, A. Rodriguez-Palacios, and F. Cominelli, "Mucosal interactions between genetics, diet, and microbiome in inflammatory bowel disease," Frontiers in Immunology, vol. 7, p. 290, 2016.

[44] K. Nishitsuji, J. Xiao, R. Nagatomo et al., "Analysis of the gut microbiome and plasma short-chain fatty acid profiles in a spontaneous mouse model of metabolic syndrome," Scientific Reports, vol. 7, no. 1, Article ID 15876, 2017.

[45] W. Scheppach, E. W. Pomare, M. Elia, and J. H. Cummings, "The contribution of the large intestine to blood acetate in man," Clinical Science, vol. 80, no. 2, pp. 177-182, 1991.

[46] E. Org, Y. Blum, S. Kasela et al., "Relationships between gut microbiota, plasma metabolites, and metabolic syndrome traits in the METSIM cohort," Genome Biology, vol. 18, no. 1, p. 70, 2017.

[47] H. Lin, The Interaction between Rat Fecal Metabonome and Microbiome during The Preobesity State, University of Chinese Academy of Sciences, 2016.

[48] H. V. Lin, A. Frassetto, E. J. Kowalik Jr. et al., "Butyrate and propionate protect against diet-induced obesity and regulate gut hormones via free fatty acid receptor 3-independent mechanisms," PLoS ONE, vol. 7, no. 4, Article ID e35240, 2012.

[49] N. Reichardt, S. H. Duncan, P. Young et al., "Phylogenetic distribution of three pathways for propionate production within the human gut microbiota," The ISME Journal, vol. 8, no. 6, pp. 1323-1335, 2014.

[50] H. M. Eid, M. L. Wright, N. V. Anil Kumar et al., "Significance of microbiota in obesity and metabolic diseases and the modulatory potential by medicinal plant and food ingredients," Frontiers in Pharmacology, vol. 8, Article ID 387, 2017.

[51] G. Den Besten, K. Van Eunen, A. K. Groen, K. Venema, D.-J. Reijngoud, and B. M. Bakker, "The role of short-chain fatty acids in the interplay between diet, gut microbiota, and host energy metabolism," Journal of Lipid Research, vol. 54, no. 9, pp. 23252340, 2013.

[52] V. K. Ridaura, J. J. Faith, F. E. Rey et al., "Gut microbiota from twins discordant for obesity modulate metabolism in mice," Science, vol. 341, no. 6150, Article ID 1241214, 2013.

[53] D. R. Donohoe, N. Garge, X. Zhang et al., "The microbiome and butyrate regulate energy metabolism and autophagy in the mammalian colon," Cell Metabolism, vol. 13, no. 5, pp. 517-526, 2011.

[54] G. Jakobsdottir, J. Xu, G. Molin, S. Ahrné, and M. Nyman, "High-fat diet reduces the formation of butyrate, but increases succinate, inflammation, liver fat and cholesterol in rats, while dietary fibre counteracts these effects," PLoS ONE, vol. 8, no. 11, Article ID e80476, 2013.

[55] H. Yadav, J. H. Lee, J. Lloyd, P. Walter, and S. G. Rane, "Beneficial metabolic effects of a probiotic via butyrate-induced GLP-1 
hormone secretion," The Journal of Biological Chemistry, vol. 288, no. 35, pp. 25088-25097, 2013.

[56] B. S. Samuel, A. Shaito, T. Motoike et al., "Effects of the gut microbiota on host adiposity are modulated by the shortchain fatty-acid binding G protein-coupled receptor, Gpr41," Proceedings of the National Acadamy of Sciences of the United States of America, vol. 105, no. 43, pp. 16767-16772, 2008.

[57] F. Imamura, S. J. Sharp, A. Koulman et al., "A combination of plasma phospholipid fatty acids and its association with incidence of type 2 diabetes: The EPIC-InterAct case-cohort study," PLoS Medicine, vol. 14, no. 10, Article ID e1002409, 2017.

[58] P. Silva Figueiredo, A. Carla Inada, G. Marcelino et al., "Fatty acids consumption: the role metabolic aspects involved in obesity and its associated disorders," Nutrients, vol. 9, no. 10, p. 1158, 2017.

[59] J. Rodríguez-Carrio, N. Salazar, A. Margolles et al., "Free fatty acids profiles are related to gut microbiota signatures and shortchain fatty acids," Frontiers in Immunology, vol. 8, p. 823, 2017.

[60] K. Chien, C. Chao, C. Kuo et al., "Plasma fatty acids and the risk of metabolic syndrome in ethnic Chinese adults in Taiwan," Lipids in Health and Disease, vol. 10, no. 1, p. 33, 2011.

[61] J. Alcock and H. C. Lin, "Fatty acids from diet and microbiota regulate energy metabolism," F1000Research, vol. 4, p. 738, 2015.

[62] M. J. Khan, K. Gerasimidis, C. A. Edwards, and M. G. Shaikh, "Role of gut microbiota in the aetiology of obesity: proposed mechanisms and review of the literature," Journal of Obesity, vol. 2016, Article ID 7353642, 27 pages, 2016.

[63] T. Li and J. Y. L. Chiang, "Bile acid signaling in metabolic disease and drug therapy," Pharmacological Reviews, vol. 66, no. 4, pp. 948-983, 2014.

[64] J. M. Ridlon, D.-J. Kang, P. B. Hylemon et al., "Bile salt biotransformations by human intestinal bacteria," Journal of Lipid Research, vol. 47, no. 2, pp. 241-259, 2006.

[65] X. Wang, D. Cui, X. Dai et al., "HuangQin decoction attenuates CPT-11-induced gastrointestinal toxicity by regulating bile acids metabolism homeostasis," Frontiers in Pharmacology, vol. 8, p. 156, 2017.

[66] C. J. Sinal, M. Tohkin, M. Miyata, J. M. Ward, G. Lambert, and F. J. Gonzalez, "Targeted disruption of the nuclear receptor FXR/BAR impairs bile acid and lipid homeostasis," Cell, vol. 102, no. 6, pp. 731-744, 2000.

[67] S. Fang, J. M. Suh, S. M. Reilly et al., "Intestinal FXR agonism promotes adipose tissue browning and reduces obesity and insulin resistance," Nature Medicine, vol. 21, no. 2, pp. 159-165, 2015.

[68] C. Thomas, A. Gioiello, L. Noriega et al., "TGR5-mediated bile acid sensing controls glucose homeostasis," Cell Metabolism, vol. 10, no. 3, pp. 167-177, 2009.

[69] M. Palau-Rodriguez, S. Tulipani, M. I. Queipo-Ortuño, M. Urpi-Sarda, F. J. Tinahones, and C. Andres-Lacueva, "Metabolomic insights into the intricate gut microbial-host interaction in the development of obesity and type 2 diabetes," Frontiers in Microbiology, vol. 6, article 1151, 2015.

[70] P. Gérard, "Metabolism of cholesterol and bile acids by the gut microbiota," Pathogens, vol. 3, no. 1, pp. 14-24, 2014.

[71] C. D. Klaassen and J. Y. Cui, "Review: mechanisms of how the intestinal microbiota alters the effects of drugs and bile acids," Drug Metabolism and Disposition: The Biological Fate of Chemicals, vol. 43, no. 10, pp. 1505-1521, 2015.

[72] J. M. Ridlon, D. J. Kang, P. B. Hylemon, and J. S. Bajaj, "Bile acids and the gut microbiome," Current Opinion in Gastroenterology, vol. 30, no. 3, pp. 332-338, 2014.
[73] G. Clarke, R. M. Stilling, P. J. Kennedy, C. Stanton, J. F. Cryan, and T. G. Dinan, "Minireview: Gut microbiota: The neglected endocrine organ," Molecular Endocrinology, vol. 28, no. 8, pp. 1221-1238, 2014.

[74] C. Thomas, R. Pellicciari, M. Pruzanski, J. Auwerx, and K. Schoonjans, "Targeting bile-acid signalling for metabolic diseases," Nature Reviews Drug Discovery, vol. 7, no. 8, pp. 678-693, 2008.

[75] H. Lin, Y. An, H. Tang et al., "Alterations of bile acids and gut microbiota in obesity induced by high fat diet in rat model," Journal of Agricultural and Food Chemistry, vol. 67, no. 13, pp. 3624-3632, 2019.

[76] Y. Zhao, J. Wu, J. V. Li, N.-Y. Zhou, H. Tang, and Y. Wang, "Gut microbiota composition modifies fecal metabolic profiles in mice," Journal of Proteome Research, vol. 12, no. 6, pp. 29872999, 2013.

[77] K. A. Romano, E. I. Vivas, D. Amador-Noguez et al., "Intestinal microbiota composition modulates choline bioavailability from diet and accumulation of the proatherogenic metabolite trimethylamine-N-oxide," mBio, vol. 6, no. 2, Article ID e02481, 2015.

[78] K. D. Corbin and S. H. Zeisel, "Choline metabolism provides novel insights into nonalcoholic fatty liver disease and its progression," Current Opinion in Gastroenterology, vol. 28, no. 2, pp. 159-165, 2012.

[79] X. Zhang, Y. Wang, F. Hao et al., "Human serum metabonomic analysis reveals progression axes for glucose intolerance and insulin resistance statuses," Journal of Proteome Research, vol. 8, no. 11, pp. 5188-5195, 2009.

[80] A. M. Asatoor and M. L. Simeshoff, "The origin of urinary dimethylamine," BBA - General Subjects, vol. 111, no. 2, pp. 384392, 1965.

[81] X. Gao, X. Liu, J. Xu, C. Xue, Y. Xue, and Y. Wang, "Dietary trimethylamine $\mathrm{N}$-oxide exacerbates impaired glucose tolerance in mice fed a high fat diet," Journal of Bioscience and Bioengineering, vol. 118, no. 4, pp. 476-481, 2014.

[82] W. Zhu, J. C. Gregory, E. Org et al., "Gut microbial metabolite TMAO enhances platelet hyperreactivity and thrombosis risk," Cell, vol. 165, no. 1, pp. 111-124, 2016.

[83] Z. Wang, A. B. Roberts, J. A. Buffa et al., "Non-lethal inhibition of gut microbial trimethylamine production for the treatment of atherosclerosis," Cell, vol. 163, no. 7, pp. 1585-1595, 2015.

[84] E. P. J. G. Neis, C. H. C. Dejong, and S. S. Rensen, "The role of microbial amino acid metabolism in host metabolism," Nutrients, vol. 7, no. 4, pp. 2930-2946, 2015.

[85] M. Holeček, "Relation between glutamine, branched-chain amino acids, and protein metabolism," Nutrition Journal, vol. 18, no. 2, pp. 130-133, 2002.

[86] C. C. Lee, S. M. Watkins, C. Lorenzo et al., "Branched-chain amino acids and insulin metabolism: The Insulin Resistance Atherosclerosis Study (IRAS)," Diabetes Care, vol. 39, no. 4, pp. 582-588, 2016.

[87] M. Doi, I. Yamaoka, M. Nakayama, K. Sugahara, and F. Yoshizawa, "Hypoglycemic effect of isoleucine involves increased muscle glucose uptake and whole body glucose oxidation and decreased hepatic gluconeogenesis," American Journal of Physiology-Endocrinology and Metabolism, vol. 292, no. 6, pp. E1683-E1693, 2007.

[88] H. Zeng, R. Tong, W. Tong et al., "Author correction: metabolic biomarkers for prognostic prediction of pre-diabetes: results from a longitudinal cohort study," Scientific Reports, vol. 7, no. 1, Article ID 16165, 2017. 
[89] C. Takashina, I. Tsujino, T. Watanabe et al., "Associations among the plasma amino acid profile, obesity, and glucose metabolism in Japanese adults with normal glucose tolerance," Nutrition \& Metabolism, vol. 13, p. 5, 2016.

[90] T. Shao, L. Shao, H. Li, Z. Xie, Z. He, and C. Wen, "Combined signature of the fecal microbiome and metabolome in patients with gout," Frontiers in Microbiology, vol. 8, p. 268, 2017.

[91] R. Liu, J. Hong, X. Xu et al., "Gut microbiome and serum metabolome alterations in obesity and after weight-loss intervention," Nature Medicine, vol. 23, no. 7, pp. 859-868, 2017.

[92] D. Il'yasova, P. Scarbrough, I. Spasojevic et al., "Urinary biomarkers of oxidative status," Clinica Chimica Acta, vol. 413, no. 19-20, pp. 1446-1453, 2012.

[93] Z.-Y. Li, L.-L. Ding, J.-M. Li et al., " ${ }^{1} \mathrm{H}-\mathrm{NMR}$ and MS based metabolomics study of the intervention effect of curcumin on hyperlipidemia mice induced by high-fat diet," PLOS ONE, vol. 10, no. 3, Article ID e0120950, 2015.

[94] X. J. Gou, Q. Tao, Q. Feng et al., "Urine metabolic profile changes of CCl.sub.4 -liver fibrosis in rats and intervention effects of Yi Guan Jian Decoction using metabonomic approach," Bmc Complementary Alternative Medicine, vol. 13, no. 1, pp. 123-123, 2013.

[95] C.-Y. Jiang, K.-M. Yang, L. Yang, Z.-X. Miao, Y.-H. Wang, and H.-B. Zhu, "A ${ }^{1} \mathrm{H}$ NMR-based metabonomic investigation of time-related metabolic trajectories of the plasma, urine and liver extracts of hyperlipidemic hamsters," PLoS ONE, vol. 8, no. 6, Article ID e66786, 2013.

[96] D. P. Patel, K. W. Krausz, C. Xie et al., "Metabolic profiling by gas chromatography-mass spectrometry of energy metabolism in high-fat diet-fed obese mice," PLoS ONE, vol. 12, no. 5, p. e0177953, 2017.

[97] I. Hwang, Y. J. Park, Y. R. Kim et al., "Alteration of gut microbiota by vancomycin and bacitracin improves insulin resistance via glucagon-like peptide 1 in diet-induced obesity," FASEB Journal, vol. 29, no. 6, pp. 2397-2411, 2015.

[98] X. Si, W. Shang, Z. Zhou et al., "Gamma-aminobutyric acid enriched rice bran diet attenuates insulin resistance and balances energy expenditure via modification of gut microbiota and short-chain fatty acids," Journal of Agricultural and Food Chemistry, vol. 66, no. 4, pp. 881-890, 2018.

[99] J. Liu, Y. Li, P. Yang et al., "Gypenosides reduced the risk of overweight and insulin resistance in C57BL/6J mice through modulating adipose thermogenesis and gut microbiota," Journal of Agricultural and Food Chemistry, vol. 65, no. 42, pp. 92379246, 2017.

[100] X. Li, E. Wang, B. Yin et al., "Effects of lactobacillus casei CCFM419 on insulin resistance and gut microbiota in type 2 diabetic mice," Beneficial Microbes, vol. 8, no. 3, pp. 421-432, 2017.

[101] H. Ma, K. He, J. Zhu, X. Li, and X. Ye, “The anti-hyperglycemia effects of Rhizoma Coptidis alkaloids: A systematic review of modern pharmacological studies of the traditional herbal medicine," Fitoterapia, vol. 134, pp. 210-220, 2019.

[102] X. Wei, J. Tao, S. Xiao et al., "Xiexin Tang improves the symptom of type 2 diabetic rats by modulation of the gut microbiota," Scientific Reports, vol. 8, no. 1, p. 3685, 2018.

[103] Y. Zhang, K. Tang, Y. Deng et al., "Effects of shenling baizhu powder herbal formula on intestinal microbiota in high-fat dietinduced NAFLD rats," Biomedicine \& Pharmacotherapy, vol. 102, pp. 1025-1036, 2018.

[104] J. Liu, S. Yue, Z. Yang et al., "Oral hydroxysafflor yellow A reduces obesity in mice by modulating the gut microbiota and serum metabolism," Pharmacological Research, vol. 134, pp. 4050, 2018.

[105] M. Chen, Z. Liao, B. Lu et al., "Huang-Lian-Jie-Du-decoction ameliorates hyperglycemia and insulin resistant in association with gut microbiota modulation," Frontiers in Microbiology, vol. 9, p. 2380, 2018.

[106] Y. Cao, G. Yao, Y. Sheng et al., "JinQi Jiangtang tablet regulates gut microbiota and improve insulin sensitivity in type 2 diabetes mice," Journal of Diabetes Research, vol. 2019, Article ID 1872134, 12 pages, 2019.

[107] S. Sun, K. Wang, K. Ma et al., "An insoluble polysaccharide from the sclerotium of Poria cocos improves hyperglycemia, hyperlipidemia and hepatic steatosis in ob/ob mice via modulation of gut microbiota," Chinese Journal of Natural Medicines, vol. 17, no. 1, pp. 3-14, 2019.

[108] M.-L. Chen, L. Yi, Y. Zhang et al., "Resveratrol attenuates trimethylamine-N-oxide (TMAO)-induced atherosclerosis by regulating TMAO synthesis and bile acid metabolism via remodeling of the gut microbiota," $m B i o$, vol. 7, no. 2, Article ID e02210-15, pp. e02210-e02215, 2016.

[109] L. M. Du, A Biology Research on The Mechanism That Gypenosides Regulates Lipids Homeostasis via Nuclear Receptor FXRMediated Pathways, Zunyi Medical College, 2018.

[110] Y. M. You, W. J. Ren, Q. Q. Liu et al., "Effects of active ingredients of Zanthoxylum oleoresin on lipid metabolism in rats fed high fat diet," Acta Nutrimenta Sinica, 2015.

[111] L. Sheng, P. K. Jena, H. Liu et al., "Obesity treatment by epigallocatechin-3-gallate-regulated bile acid signaling and its enriched Akkermansia muciniphila," FASEB Journal, vol. 32, no. 12, Article ID fj.201800370R, pp. 6371-6384, 2018.

[112] T. Hampton, "Ongoing research points to key role of gut microbes in cardiovascular health," Circulation, vol. 134, no. 21, pp. 1687-1688, 2016.

[113] M. Li, X. Shu, H. Xu et al., "Integrative analysis of metabolome and gut microbiota in diet-induced hyperlipidemic rats treated with berberine compounds," Journal of Translational Medicine, vol. 14, no. 1, article no. 237, 2016.

[114] L. Wang, Z. Pi, S. Liu, Z. Liu, and F. Song, "Targeted metabolome profiling by dual-probe microdialysis sampling and treatment using Gardenia jasminoides for rats with type 2 diabetes," Scientific Reports, vol. 7, no. 1, Article ID 10105, 2017.

[115] K. Gao, R. Yang, J. Zhang et al., "Effects of Qijian mixture on type 2 diabetes assessed by metabonomics, gut microbiota and network pharmacology," Pharmacological Research, vol. 130, pp. 93-109, 2018. 


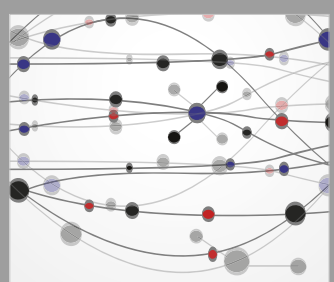

The Scientific World Journal
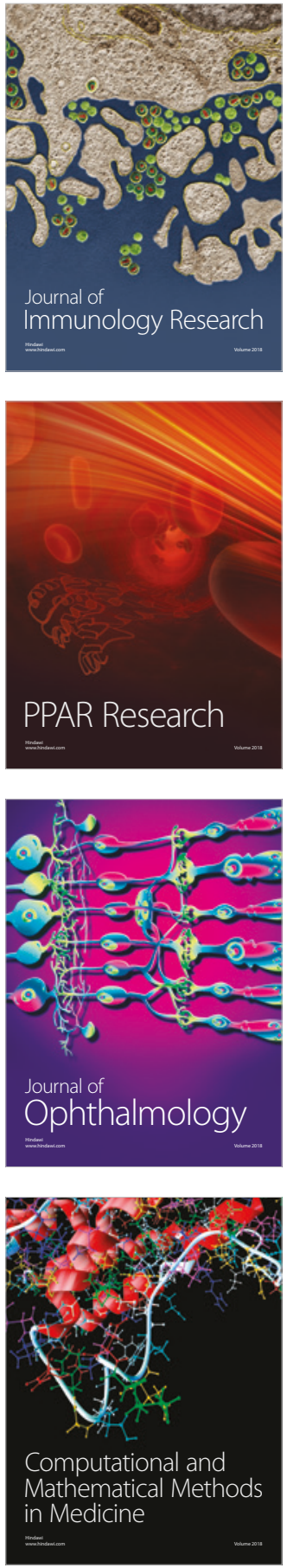

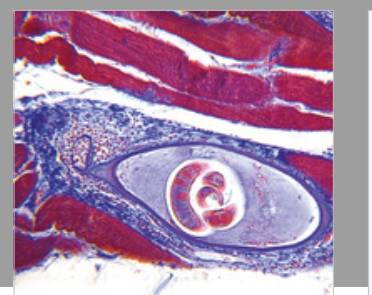

Gastroenterology Research and Practice

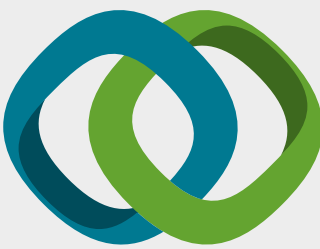

\section{Hindawi}

Submit your manuscripts at

www.hindawi.com
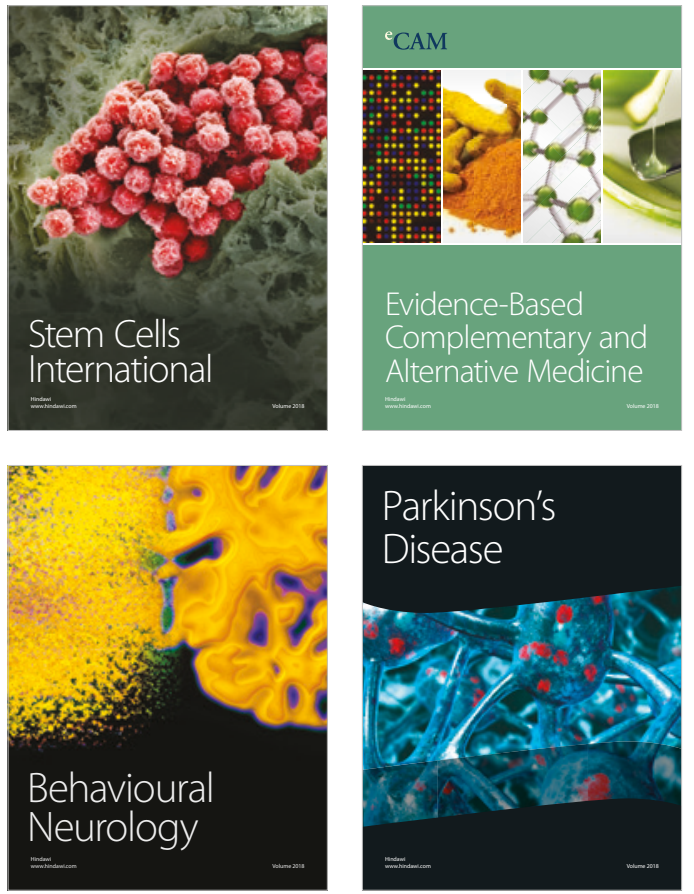

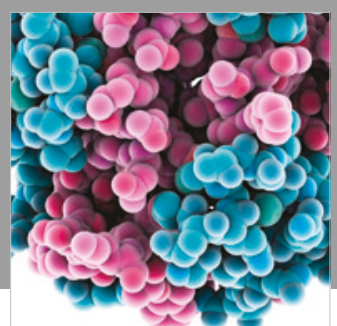

ournal of

Diabetes Research

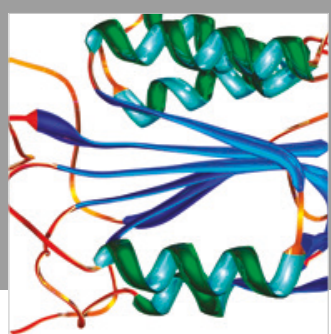

Disease Markers
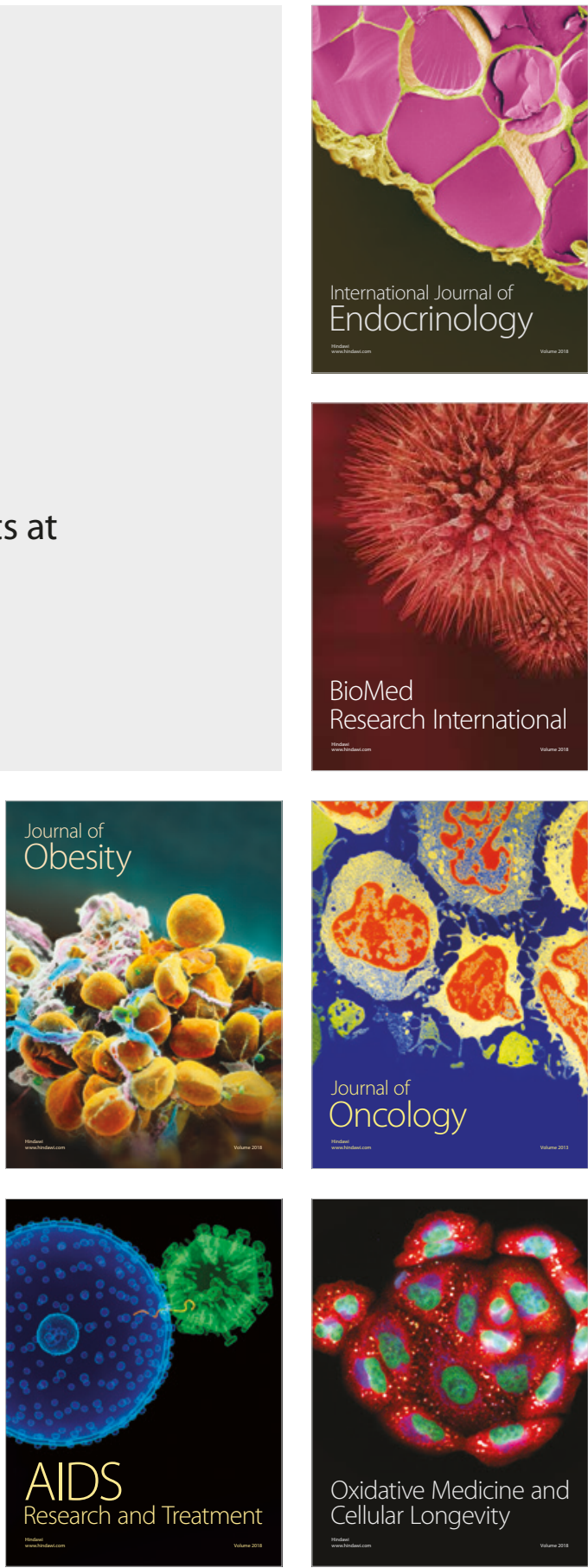